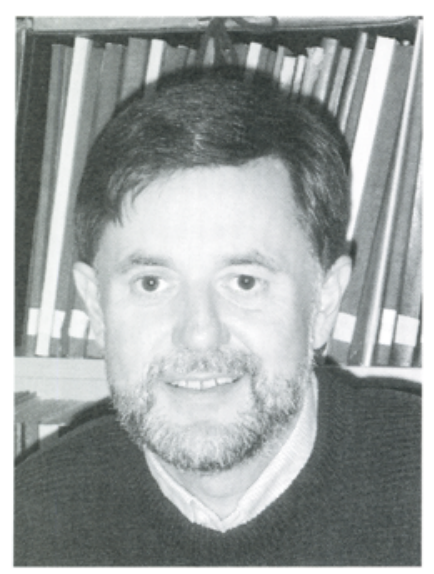

Reinhard Zölitz-Möller Gasteditor dieses Heftes

\section{Kartographie von Umweltproblemen}

Der Schutz der Umwelt ist gerade in den die natürlichen Ressourcen oft wenig schonenden Industriegesellschaften eine wichtige Aufgabe, die zu bewältigen auch die moderne raumbezogene Informationsverarbeitung mithelfen muss und kann. Wenn es dabei um die Kommunikation von Umweltzuständen und Entscheidungsunterstützung geht, sind die Produkte der Kartographie essenziell. Wie die Aufgaben des Umweltmanagements, der Umweltforschung und der Umweltinformation durch die Kartographie unterstütz† werden, soll dieses Heft der KN zeigen. Die Beiträge dokumentieren je auf ihre Weise, wie moderne Methoden und die Integration geeigneter Geodaten dazu beitragen können.

Jörg Hartleib zeigt, wie eine um Nachhaltigkeit bemühte Waldwirtschaft durch eine Forst-GIS-Anwendung in die Lage versetzt wird, die richtigen Entscheidungen zu treffen. Dabei werden, wie heute bei vielen anderen umweltbezogenen Aufgaben auch, Geofachdaten und amtliche Geobasisdaten in einem möglichst nutzerfreundlichen Informationssystem integriert. Es erweist sich immer wieder, dass diese Integration keine Knopfdruck-Aufgabe ist und der Weg vom Desktop-GIS bis zur "Karte" nach wie vor besondere Anstrengungen erfordert.

Auch im Beitrag von Görres Grenzdörffer und Ralf Bill dient Datenintegration einer Umweltmanagementaufgabe. Für die Ausweisung von potenziellen Überschwemmungsgebieten müssen hochgenave, aktuelle und vollständige Geländehöhendaten Ihier mit Hilfe des Laserscanningl erfasst und mit amtlichen Liegenschaftskarten verknüpft werden. Auch hier stehen am Ende wieder analoge Karten, die in einen Verwaltungsvorgang mit Bürgerbeteiligung einfließen müssen. Die Kartographie über- nimmt dabei unabdingbare Visualisierungsaufgaben.

Bei der Visualisierung werden Daten in ein sichtbares Bild transformiert.

Wie dies heute im Kontext eines Landschaftsinformationssystems zur Unterstützung der Erkundung und der Erklärung landschaftlicher Zusammenhänge erfolgen kann, zeigt der Beitrag von Uwe Heinrich über die im Zentrum für Agrarlandschaft und Landnutzungsforschung (ZALF) eingesetzten Techniken. Gestuft nach Nutzerprofilen kommen hier WebGIS, Multimedia- und klassische GIS-Techniken zum Einsatz. Die je nach Bedarf unterschiedliche, aber stets aktuell erzeugte Bildschirmkarte ist hier das Produkt, welches Datenrecherche, Präsentation und Auswertung für die Mitarbeiter der Forschungseinrichtung unterstützt.

Breitere Nutzerschichten haben Jens Fitzke und Klaus Greve im Blick, wenn sie über die Visualisierungsaufgaben von Umweltinformationssystemen berichten. Dabei gehen sie auf den aktuellen Stand bei WebMap-Diensten auf Grundlage der openGIS-Spezifikationen ein. Auch in diesem Beitrag wird die zentrale Bedeutung der Karte für die Kommunikation von Umweltinformation deutlich.

Die Beiträge zeigen, dass die Umweltkartographie ein aktuelles und trotz rasanter technischer Weiterentwicklung stets auch individuelle Lösungen erforderndes und damit spannendes Feld für Kartographen und Geowissenschaftler ist. In der Hoffnung, dass wir auf diese Weise mithelfen, unseren Enkeln eine lebenswerte Welt zu erhalten, grüßt Sie als Gastherausgeber

Reinhard Zölitz-Möller

Unserem Gasteditor zu diesem Heft dankt die Schriftleitung der KN recht herzlich für seine Tätigkeit. Kennen Sie schon die neve Internetseite der DGFK? http://www.dgfk.net/ 